\title{
The Motion of Polymer Chains in Solutions. I. Reorientational Motion of Phenyl Groups of Polystyrene Chains in Solutions of Carbon Tetrachloride by Raman Line Shape Analysis
}

\author{
Hiroyasu Nomura and Yutaka Miyahara \\ Department of Chemical Engineering, Faculty of Engineering, \\ Nagoya University, Chikusa-ku, Nagoya 464, Japan.
}

(Received April 18, 1975)

\begin{abstract}
The reorientational correlation time in carbon tetrachloride of the phenyl groups of polystyrene was measured by Raman-line shape analysis. Up to the point where the molecular weight of the polymer reaches about $1.0 \times 10^{4}$, the larger the molecular weight, the longer is the reorientational correlation time of the phenyl groups. For molecular weights above $1.0 \times 10^{4}$, the reorientational correlation time of the phenyl groups levels off at about $4 \times 10^{-10} \mathrm{sec}$. The phenyl groups of the polystyrene chains rotate about 130 times more slowly than the molecules of toluene in carbon tetrachloride.
\end{abstract}

KEY WORDS Molecular Motion / Raman / Reorientational Correlation Time / Polystyrene

In a previous paper, ${ }^{1}$ we reported that the ultrasonic absorption in solutions of polymers in various solvents shows a relaxation behavior in the megahertz frequency range. The plot of $\Delta \alpha / f^{2}\left(\Delta \alpha=\alpha-\alpha_{0}\right.$, where $\alpha$ and $\alpha_{0}$ denote the absorption coefficients of the solution and pure solvent, respectively) against the frequency shows a relaxation curve having a single or double relaxation time in all cases. We considered that this relaxation is due to the local rotation of polymer chain segments in the solutions and estimated the energy difference and activation energy from the temperature changes of the absorption on the basis of 2-state model.

However, the limiting value of $\left(\Delta \alpha / f^{2}\right)$ at the $f \rightarrow \infty$ is not equal to the value calculated from the shear viscosity at the high frequency, $\eta_{\infty}$, for all polymer solutions. This fact suggests that there are more than one absorption mechanism due either to the rotation of the segment or to the vibrational mode of polymer chains in solutions.

In Raman spectroscopic studies of the liquid state molecular dynamics, the most important quantity obtainable is the reorientational correlation time $\tau_{\text {or. }}$. When the molecule is a symmetric top, the analysis of isotropic and anisotropic Raman-band shapes of nondegenerated vibrations uniquely determines the reorientational correlation time for the tumbling motion of the molecules.

The reorientational correlation times in carbon tetrachloride of phenyl groups of polystyrene were estimated from the Raman-line shape $\left(1002 \mathrm{~cm}^{-1}\right)$ analysis. The dependence of the reorientational correlation time on the molecular weight was discussed in comparing these values with the NMR relaxation data in polymer solutions. ${ }^{2,3}$

\section{EXPERIMENTAL}

An argon ion laser (Coherent Radiation, QW) was used for light source excited at $4880 \AA$ in all measurements. The scattered light was analyzed with a double monochrometer (JRS-U1 Laser Raman Spectrometer).

The polystyrene samples used were the monodispersed standard polystyrene (Pressure Chem. Co.) and these molecular weights were 600 $\left(M_{w} / M_{n} \ll 1.10\right), 4.0 \times 10^{3}(1.10), 5.3 \times 10^{4}(1.06)$ and $2.0 \times 10^{5}$. The concentration of polymer solutions was below $10 \%$ and Raman lines of polymer solutions were measured at room temperature. 
The Motion of Polymer Chains in Solutions. I.

\section{RAMAN THEORY AND ANALYSIS}

Analysis of the Raman-line shape of the $\nu_{2}\left(a_{1 \mathrm{~g}}\right)$ fundamental in polystyrene in solution yields information on the rotational relaxation times of the phenyl groups. The theory has recently been summarized ${ }^{4}$ and only needs a cursory introduction here. The mechanics of the data aquisition and analysis require some clarification.

All experiments were done using $90^{\circ}$ scattering and linearly polarized incident light. With $I_{\|}(\omega)$ and $I_{\perp}(\omega)$ representing the strong and weak component of the scattered light, one can write

$$
\begin{gathered}
I_{\|}(\omega)=I_{\text {isot }}(\omega)+\frac{4}{3} I_{\mathrm{anis}}(\omega) \\
I_{\perp}(\omega)=I_{\mathrm{anis}}(\omega) \\
\rho_{\mathrm{s}}=\frac{I_{\perp}(\omega)}{I_{\| l}(\omega)}=\frac{I_{\mathrm{anis}}(\omega)}{I_{\text {isot }}(\omega)+\frac{4}{3} I_{\mathrm{anis}}(\omega)}
\end{gathered}
$$

where $\rho_{\mathrm{s}}$ is the depolarization ratio. $I_{\text {isot }}(\omega)$ represents the intrinsic vibration line shape whereas $I_{\mathrm{anis}}(\omega)$ is a convolution of the vibrational line shape and the reorientational spectrum. The problem is to separate the two accurately.

$I_{\text {isot }}(\omega)$ can be extracted directly from measurements of $I_{\|}(\omega)$ and $I_{\perp}(\omega)$. However, since the depolarization ratio is very small for the band under consideration, the intrinsic vibration line shape $I_{\text {isot }}(\omega)$ and the measured line shape $I_{\|}(\omega)$ can be considered to be the same without introduction of significant errors. Generally however, the measured spectrum is a convolution of the true spectrum and Gaussian slit function $S(\omega)$. One can write

$$
I_{\mathrm{anis}}^{\prime}(\omega)=\int_{-\infty}^{\infty} I_{\mathrm{or}}(\omega) I_{\mathrm{isot}}(\omega) S(\omega) \mathrm{d} \omega
$$

and

$$
I_{\mathrm{isct}}^{\prime}(\omega)=\int_{-\infty}^{\infty} I_{\mathrm{isot}}(\omega) S(\omega) \mathrm{d} \omega
$$

where the primed and unprimed functions denote the measured and true shapes, respectively. At first sight, it would appear that the order of convolution is not important and hence the slit function would cancel. This is not the case except when the line widths are very similar in magnitude. To obviate the larger error associated with the procedure, true line widths were determined directly as a function of slit width and a plot is shown in Figure 1.

For the $1002-\mathrm{cm}^{-1}$ line of polystyrene, $I_{\|}(\omega)$

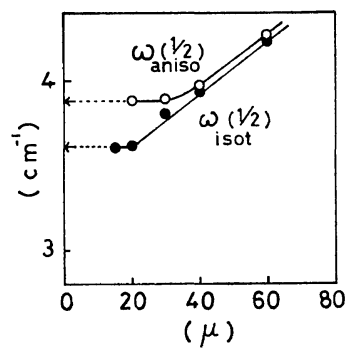

Figure 1. Line-width of $\nu_{2}$ of phenyl groups in polystyrene $(M=600)$ in carbon tetrachloride as a function of slit width.

can be determined directly by setting the slit to $20 \mu$ or less. In order to obtain $I_{\text {isot }}(\omega), I_{\perp}(\omega)$ must be evaluated at the same slit width. Examples of measured $I_{\|}(\omega)$ and $I_{\perp}(\omega)$ are shown in Figure 2. Errors arising from monochrometer polarization response, beam convergence, light collection angles, analyzer light leak, ellipticity of the excited light polarization, laser power fluctuations, and stray light, have all been minimized.

Implicit in the treatment of data are the assumptions that $I_{\text {isot }}(\omega)$ and $I_{\text {anis }}(\omega)$ are Lorentzian and that vibrational and reorientational motions are not coupled. A test of those assumptions is shown in Figure 3 in which $I_{\text {isot }}(\omega)$ and $I_{\text {anis }}(\omega)$ normalized to unit height, are displayed (point).

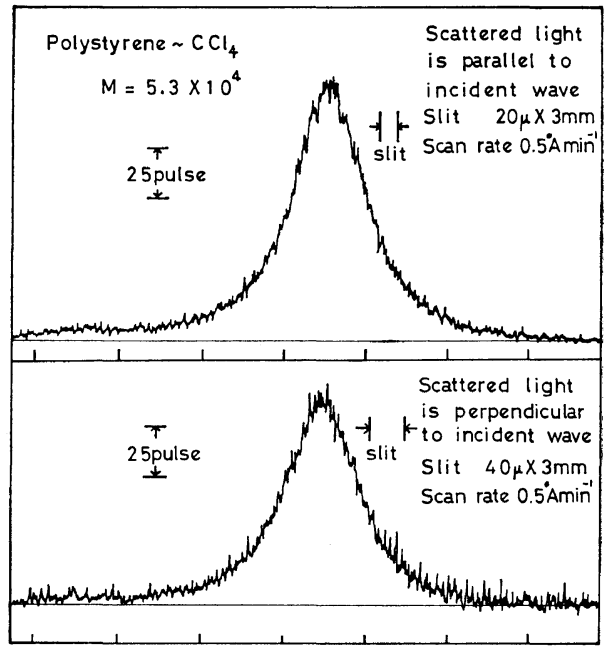

Figure 2. (A) Polarized Raman spectrum, $I_{\|}(\omega)$, (B) depolarized Raman spectrum, $I_{\perp}(\omega)$ of $\nu_{2}$ of phenyl groups in polystyrene $\left(M=5.3 \times 10^{4}\right)$ in carbon tetrachloride. 


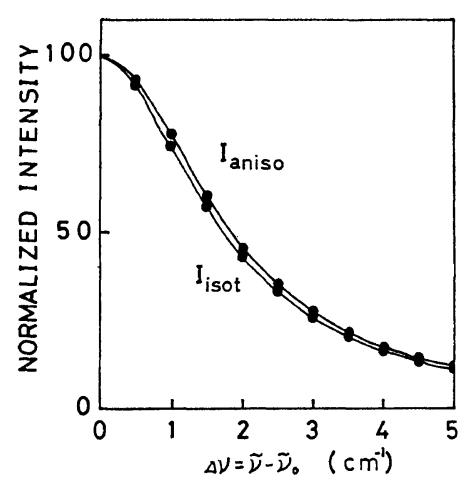

Figure 3. Normalized line shaps of $I_{\text {isot }}(\omega)$ and $I_{\text {anis }}(\omega)$ of the $\nu_{2}$ band of phenyl groups in polystyrene $\left(M=5.3 \times 10^{4}\right)$ in carbon tetrachloride.

The solid lines are the calculated Lorentz function based on the observed line width. Because $I_{\text {isot }}(\omega)$ and $I_{\text {anis }}(\omega)$ are Lorentzian and because $I_{\mathrm{anis}}(\omega)$ is a convolution of $I_{\text {isot }}(\omega)$ with $I_{\mathrm{or}}(\omega)$, the $I_{\mathrm{or}}(\omega)$ is also Lorentzian. Accordingly,

$$
\omega_{1 / 2}(\text { anis })=\omega_{1 / 2}(\text { isot })+\omega(\text { or })
$$

where the $\omega_{1 / 2}$ terms are semihalf width. Furthermore, the reorientational correlation time is obtained as follows.

$$
\tau^{-1} \text { (or) }=2 \pi C \omega \text { (or) }
$$

where $C$ is the velocity of light.

\section{RESULTS AND DISCUSSION}

The plots of $\omega_{\|}\left(\frac{1}{2}\right)$ and $\omega_{\perp}\left(\frac{1}{2}\right)$ against slit widths for toluene, dibenzyl and polystyrene solutions in carbon tetrachloride were the same as shown in Figure 1. The measured line shapes of $\nu_{2}\left(a_{1 \mathrm{~g}}\right)$ of phenyl groups in solutions were also of the Lorentzian type, as in Figure 3. The Raman frequencies, the depolarization ratio, $\omega_{\|}\left(\frac{1}{2}\right)$ and $\omega_{\perp}\left(\frac{1}{2}\right)$ at the $\nu_{2}\left(a_{1 \mathrm{~g}}\right)$ of phenyl groups in solutions were summarized in Table I. Relations between the Raman frequencies $\nu_{2}\left(a_{1 \mathrm{~g}}\right)$ of phenyl groups in solutions and its depolarization ratio and the molecular weight of polymers are shown in Figure 4 and the relation of $\omega_{\|}\left(\frac{1}{2}\right)$ and $\omega_{\perp}\left(\frac{1}{2}\right)$ to the molecular weight of polymers is shown in Figure 5.

As is seen in Figures 4 and 5 , the relations between $\nu_{2}\left(a_{1 \mathrm{~g}}\right), \quad \rho_{\mathrm{s}}, \quad \omega_{\|}\left(\frac{1}{2}\right)$ and $\omega_{\perp}\left(\frac{1}{2}\right)$ and $\log M$ for toluene and dibenzyl are different from those of the polymer solution in carbon tetrachloride. Furthermore, the difference between $\omega_{\|}\left(\frac{1}{2}\right)$ and $\omega_{\perp}\left(\frac{1}{2}\right)$, that is, $\omega_{\text {or }}\left(\frac{1}{2}\right)$, decreases with increasing molecular weight of polymer and tends to be constant. The values of $\tau$ (or) for toluene, dibenzyl and polystyrenes in solutions of carbon tetrachloride are shown in the 7th column of Table I. In other words, the reorientational correlation time, $\tau_{\text {or }}$, increases with increasing molecular weight of polymers in solution.

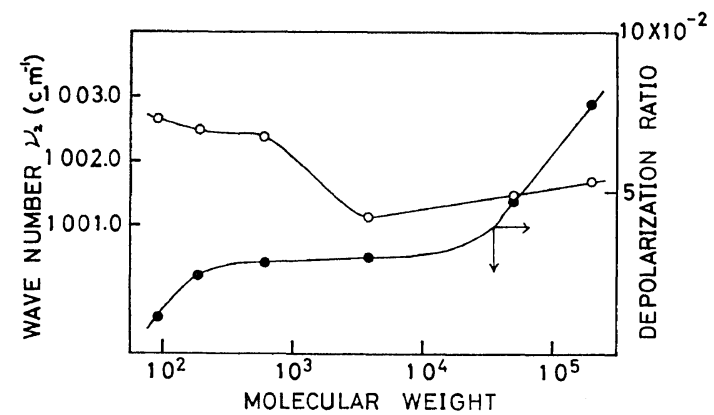

Figure 4. Dependence of Raman frequencies, $\nu_{2}$, and depolarization ratio of the phenyl groups in polystyrene in carbon tetrachloride on molecular weight of polymer.

Table I. Raman frequencies, depolarization ratio, $\omega_{\text {isot }}\left(\frac{1}{2}\right), \omega_{\text {anis }}\left(\frac{1}{2}\right), \omega_{\circ}(\omega)$, and reorientational correlation times of phenyl groups in various samples

\begin{tabular}{lrrccccc}
\hline & $\begin{array}{c}\text { Molecular } \\
\text { weight }\end{array}$ & $\begin{array}{c}\nu_{2}\left(a_{1 \mathrm{~g}}\right), \\
\mathrm{cm}^{-1}\end{array}$ & $\begin{array}{c}\text { Depolarization } \\
\text { ratio, } \times 10^{2}\end{array}$ & $\begin{array}{c}\omega_{\text {isot }}\left(\frac{1}{2}\right), \\
\mathrm{cm}^{-1}\end{array}$ & $\begin{array}{c}\omega_{\text {anis }}\left(\frac{1}{2}\right), \\
\mathrm{cm}^{-1}\end{array}$ & $\begin{array}{c}\omega_{\text {or }}(\mathrm{ob}), \\
\mathrm{cm}^{-1}\end{array}$ & $\begin{array}{c}\tau_{\text {or }}, \\
\times 10^{12}\end{array}$ \\
\hline Toluene & 92 & 1002.66 & 1.12 & 1.71 & 3.56 & 1.84 & 2.88 \\
Dibenzy1 & 182 & 1002.48 & 2.44 & 2.30 & 3.70 & 1.40 & 3.79 \\
PS 1 & 600 & 1002.38 & 2.82 & 3.41 & 3.89 & 0.47 & 11.2 \\
PS 2 & 4000 & 1001.13 & 2.99 & 3.54 & 3.70 & 0.15 & 33.6 \\
PS 3 & 53000 & 1001.46 & 4.76 & 3.79 & 3.83 & 0.03 & 169 \\
PS 4 & 200000 & 1001.67 & 7.55 & 3.79 & 3.81 & 0.01 & 400 \\
\hline
\end{tabular}


The Motion of Polymer Chains in Solutions. I.

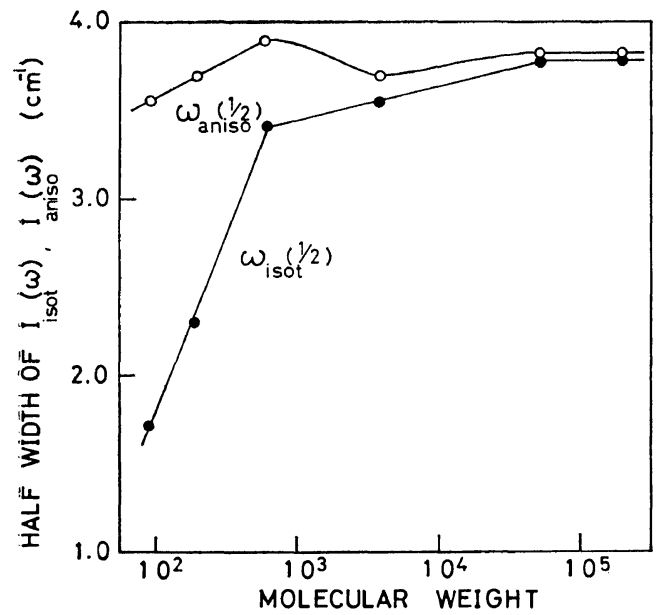

Figure 5. Dependence of $\omega_{\text {isot }}\left(\frac{1}{2}\right)$ and $\omega_{\text {anis }}\left(\frac{1}{2}\right)$ with molecular weight of polystyrene.

For NMR spectra of polystyrene in solutions of carbon tetrachloride Bovey $^{3}$ reported that, since the line width of phenyl groups of polystyrene was independent of the viscosity of polymer solutions below $50 \mathrm{wt} \%$, the intramolecular interaction of segments in the polymer chains made the dominant contribution to the spin-lattice relaxation time $\left(T_{1}\right)$ of phenyl groups rather than the intermolecular dipole-dipole interactions. Bovey, et al., ${ }^{2}$ also showed from their experimental results for the $T_{1}$ of the phenyl groups of PBG in trifluoroacetic acid and pure benzene that benzene rings on the random-coil polymer chains rotate $40-50$ times more slowly than the molecules of benzene itself.

The author's results for the reorientational correlation times of phenyl groups of polystyrene solutions in carbon tetrachloride are in good agreement with above NMR results. The larger the molecular weight, the longer is the reorientational correlation time of phenyl groups until the molecular weight of polymer becomes about $1.0 \times 10^{4}$. For the region of molecular weights above $1.0 \times 10^{4}$, the reorientational correlation time of phenyl groups of polystyrene becomes about $4 \times 10^{-10} \mathrm{sec}^{-1}$.

Since the reorientational correlation times of phenyl groups of toluene and polystyrene in solutions of carbon tetrachloride are $2.88 \times 10^{-12}$ and $4 \times 10^{-10} \mathrm{sec}^{-1}$, respectively, the phenyl groups of the polystyrene chains rotate about 130 times more slowly than those of toluene in carbon tetrachloride.

However, the difference between the $1.69 \times$ $10^{-10}$ and $4 \times 10^{-10} \mathrm{sec}^{-1}$ at the molecular weight $5.30 \times 10^{4}$ and $2.0 \times 10^{5}$, respectively, is indistinguishable for the accuracy of these experiments. Accordingly, it is suggested that the reorientational correlation time of phenyl groups in polystyrene above molecular weight of $1.0 \times$ $10^{4}$, is of the order of $10^{-10} \mathrm{sec}^{-1}$.

This molecular weight dependence is also evident in the relation between the depolarization ratio and the Raman frequency of $\nu_{2}$ mode of phenyl groups and the molecular weight.

The dependence of the reorientational correlation time on the molecular weight was considered as follows; The reorientational correlation time of the phenyl groups is related to the tumbling motion of them, and this reorientational correlation time yields information on the rotational diffusion constant which itself refers to the friction constant of the molecular motion of phenyl groups in polystyrene in solution. The larger the molecular weight is, the more the interaction between the phenyl groups in a polystyrene chain. The reorientational correlation time thus becomes longer. However in the region of sufficiently high molecular weights where the segmental distribution of polymer chains reaches a constant distribution, i.e., the Gaussian distribution, the interaction between the phenyl groups in a polystyrene chain will be constant. As a result, the reorientational correlation time becomes a constant.

Recently, Ono, et al., ${ }^{5}$ showed that there are other acoustic relaxations around $500 \mathrm{MHz}$ using Mikhailov and Safina's data for polystyrene solutions in MEK and toluene. They considered this relaxation mechanism to be due to the vibrational relaxation of the phenyl groups.

As is seen in Figure 5 and Table $I$, the reorientational correlation times of phenyl groups are about $3-4 \times 10^{-10} \mathrm{sec}^{-1}$, that is, the relaxation frequency is about $400-500 \mathrm{MHz}$. This result suggests that the ultra-high frequency relaxation in solution of polystyrene is not only due to the vibrational relaxation of the phenyl groups in the polystyrene chains but also to the rotational relaxation of them. 
H. NOMURA and Y. MiYahara

\section{REFERENCES}

1. S. Kato, H. Kondo, I. Fujio, H. Nomura, and Y. Miyahara, J. Chem. Soc. Japan, 1981 (1974).

2. F. A. Bovey, G. V. D. Ties, and G. Pillipovich, J. Polym. Sci., 38, 73 (1959).

3. F. A. Bovey, J. Chem. Phys. 32, 1887 (1960).
4. F. J. Bartoli and T. A. Litovitz, ibid., 56, 404, 413 (1973).

5. K. Ono, H. Shintani, O. Yano, and Y. Wada, Polymer J., 5, 164 (1973).

6. I. G. Mikhailov and E. B. Safina, Soviet-Phys., Acoustics, 17, 335 (1972). 